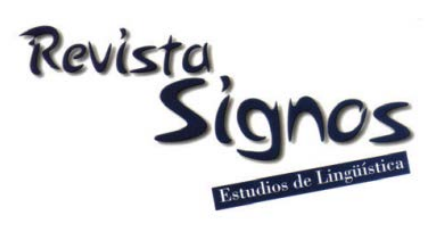

\title{
Neología sintagmática anglicada en español: Calcos y préstamos
}

\author{
Neological Anglicized Phrases in Spanish: Calques and \\ Loanwords
}

Constanza Gerding

Salas

UNIVERSIDAD DE CONCEPCIÓN

CHILE

cgerding@udec.cl

\author{
Paola Cañete \\ González \\ UNIVERSIDAD DE CONCEPCIÓN \\ CHILE \\ pcanete@udec.cl
}

\author{
Carolin Adam \\ UNIVERSIDAD DE CONCEPCIÓN \\ CHILE \\ cadam@udec.cl
}

Recibido: 17-III-2016 / Aceptado: 21-VII-2017

\section{Resumen}

Debido a la influencia lingüístico-cultural estadounidense, las unidades léxicas provenientes del inglés son una de las formas prolíficas de innovación en español, tal como lo sugieren algunos de los resultados de estudios realizados por la Antena Chilena de Neología, Universidad de Concepción, Chile. Este tipo de formación neológica se ha evidenciado en el español a través de diversas manifestaciones, a saber, los préstamos, los calcos y las creaciones híbridas. Se ha demostrado que el préstamo del inglés, particularmente, tiene una importancia significativa en la innovación léxica de distintas variantes del español (Goddard, 1980; Lorenzo 1996; Gimeno \& Gimeno, 2003; Novotná, 2007); de allí el interés por ahondar en formaciones neológicas de este origen. En el presente trabajo se aborda la presencia del inglés en el español mediante la creación de unidades léxicas sintagmáticas; para ello se consideraron los calcos del inglés y las formaciones híbridas que contienen préstamos anglicados. La muestra se obtuvo a partir de unidades sintagmáticas neológicas creadas por calco del inglés y formaciones híbridas formadas por una voz patrimonial y un préstamo anglicado recogidas en prensa chilena entre 2003 y 2014. El análisis se realizó de acuerdo a la recurrencia, la composición gramatical y los ámbitos del conocimiento en las que se utilizaban estos neologismos. Los resultados determinaron que los calcos eran más frecuentes que las formaciones híbridas, que las combinaciones sintagmáticas respondían a distintas estructuras gramaticales con predominio de los sintagmas nominales y con mayor presencia en determinadas áreas del conocimiento.

Palabras Clave: Neologismo, sintagma, préstamo del inglés, formación híbrida, calco. 


\begin{abstract}
As results from studies carried out by the Antena Chilena de Neología - Universidad de Concepción suggest, lexical units from English are one of the prolific forms of innovation in Spanish, especially due to the linguistic and cultural influence of the United States. Neological word formations of this origin, such as loanwords and loan translations from English, and hybrid word formations are evident in Spanish. Previous research has proven that English loan words are significant today in lexical innovation in different varieties of Spanish (Goddard, 1980; Lorenzo 1996; Gimeno \& Gimeno, 2003; Novotná, 2007); therefore, it was interesting to examine other types of Anglicized neological formations. This study focuses on the presence of Anglicized phrases in Spanish, i.e., loan translations from English and hybrid formations containing English loanwords. The sample was obtained from neological syntagmatic units formed either by English calque or hybrid formations consisting of a patrimonial word and an English loanword, collected from Chilean press between 2003 and 2014. The analysis was performed according to recurrence, grammatical configuration, and domains where these Anglicized neologisms were used. The results determined that loan translations were more frequent than hybrid formations, that the syntagmatic combinations consisted of a variety of grammatical structures, predominantly noun phrases, and that both types of neological units were overridingly used in specific domains.
\end{abstract}

Key Words: Neologism, syntagma, loan word, hybrid formation, calque.

\title{
INTRODUCCIÓN
}

Al igual que otras lenguas, el español actual recibe la influencia de otros idiomas y culturas, entre los que resalta el influjo de la lengua y cultura anglosajonas, cuestión que obedece principalmente al papel hegemónico de Estados Unidos en América Latina. Bernal-Meza (2008), por ejemplo, habla del reconocimiento de algunos gobiernos latinoamericanos a la "absoluta hegemonía" de Estados Unidos y de su visión sobre el orden mundial y la globalización. Por otra parte, Briceño Ruiz (2006: 433) plantea que la Unión Europea:

"no aspira a eliminar la hegemonía estadounidense en América Latina y el Caribe; nada más se propone evitar que Estados Unidos mejore su posición económica a expensa de los intereses europeos".

En este escenario, pareció interesante analizar otro aspecto de la innovación léxica del español, además de los estudiados anteriormente por la Antena Chilena de Neología. Los estudios previos abordaban los préstamos en general, por una parte, y los préstamos del inglés, por otra. Se decidió, entonces, observar la influencia del inglés desde la perspectiva de la sintagmación, es decir, unidades constituidas por calco anglicado o por la construcción híbrida de préstamos y voces patrimoniales. En este sentido, estudios como el de Novotná (2007) y el de Gómez Capuz (2009) entregan categorizaciones pormenorizadas de préstamos y calcos anglicados, sin ahondar específicamente en las formas sintagmáticas con influencia del inglés. Este estudio aportaría al análisis más allá del préstamo simple por cuanto se refiere al uso 
específico de construcciones sintagmáticas anglicadas en español, lo que ayudaría a disciplinas tales como la lexicografía, la lexicología y la semántica, que se ocupan del estudio del léxico y su posterior incorporación a diccionarios; y el periodismo y la traducción, que contribuyen a la difusión de unidades procedentes de otras lenguas.

Para este trabajo, se utilizaron los datos de la Antena Chilena de Neología, cuya base consta de aproximadamente 20.000 piezas neológicas documentadas. Los sintagmas anglicados fueron extraídos de esta base mediante el procedimiento de filtración, con el propósito de analizarlos desde el punto de vista de los tipos y subtipos de formación, la estructura de los sintagmas, las áreas temáticas en las que se encontraron y las marcas tipográficas con las que se suelen etiquetar.

\section{Marco teórico}

El presente estudio forma parte de las investigaciones realizadas por la Antena Chilena de Neología, nodo Universidad de Concepción ${ }^{1}$, integrante de la red Antenas Neológicas, que es un proyecto internacional coordinado por el Observatori de Neologia (en adelante, OBNEO) del Institut Universitari de Lingüística Aplicada de la Universitat Pompeu Fabra de Barcelona. El objetivo central de esta red es la observación y el estudio de la innovación léxica del español con el propósito de contribuir a la ampliación descriptiva de las distintas variedades, difundir las nuevas creaciones léxicas y aportar a la actualización lexicográfica.

En este marco y gracias a la observación de las distintas formas de creación léxica en la prensa por más de una década, el nodo Universidad de Concepción de la Antena Chilena de Neología ha realizado, entre otros estudios, análisis de la influencia anglosajona en el español (Gerding, Fuentes \& Kotz, 2012; Fuentes, Gerding, Gómez \& Kotz, 2012; Gerding, Fuentes, Gómez \& Kotz, 2014). En esta misma línea investigativa, para esta investigación la exploración se ha centrado en la neología léxica en construcciones sintagmáticas formadas por calco y con préstamo anglicados.

En la actualidad, la influencia lingüístico-cultural del inglés es incuestionable en muchos países del mundo. Esta propensión se explica por la fuerte presencia de Estados Unidos en ámbitos como la economía, la cultura, el poderío militar, la política y la ciencia (Gerding et al., 2012), no quedando ajeno a ella el uso de la lengua, que en español se manifiesta con la incorporación de diversos tipos de unidades léxicas formadas con elementos anglicados, que permiten nombrar realidades nuevas o renombrar realidades existentes.

Estas formas anglicadas aparecen por diversos motivos: necesidad de llenar vacíos denominativos, premura en la adopción de voces foráneas ante cambios vertiginosos, desterminologización de unidades originadas en ámbitos especializados, economía lingüística, prestigio atribuido a la lengua de origen, desconocimiento de la existencia 
de un equivalente en la lengua receptora, contacto entre lenguas y culturas, eficacia u originalidad en la comunicación y otras razones estilísticas, por nombrar las más esgrimidas (Deroy, 1971; Mounin, 1990; Cabré, 1993; Alvar Ezquerra, 1999; Diéguez, 2004; Gerding et al., 2012, 2014; Guerrero Ramos, 2013). En el caso de la variante chilena, como en el de otras, estos motivos están relacionados con determinados factores sociopolíticos que propician la adopción de este tipo de neologismos, tales como el modelo neoliberal de economía de mercado con base en paradigmas estadounidenses, la gran apertura de la actividad comercial y económica al mundo globalizado, los avances de la ciencia y la tecnología en el mundo y el inglés como lengua de prestigio.

En este contexto, varios autores (Domínguez Mejías, 2002; Delgado Álvarez, 2005; Gerding et al., 2012, 2014) destacan la importancia de los medios de comunicación y el rol de los periodistas como potenciadores del uso de neologismos anglicados. Autores como Alvar Ezquerra (1998, 2007), Romero Gualda (1999), Ortega Martín (2001), Perdiguero (2003), Gerding et al. (2014) sostienen que los medios de comunicación constituyen un excelente observatorio para verificar la aparición y propagación de neologismos. En efecto, al lenguaje periodístico se le describe como un surtidor inagotable de ejemplos que permiten ilustrar los procesos de formación de palabras e incorporación de voces provenientes de otros idiomas y también como testimonio de la vitalidad de una lengua. Ahora bien, dada la capacidad divulgadora de palabras nuevas de la prensa, resulta interesante estudiar las unidades léxicas anglicadas en este tipo de fuente para observar el comportamiento y las costumbres de la sociedad y para examinar la influencia de unas culturas en otras. De hecho, se ha documentado ampliamente la presencia de voces y expresiones provenientes del inglés en el lenguaje periodístico en lengua española (Ortega Martín, 2001; Diéguez, 2004; Delgado Álvarez, 2005; Sáez Godoy, 2005; Morin, 2006; Esteban Asencio, 2008; Oncins-Martínez, 2009; Fuentes et al., 2012; Gerding et al., 2012, 2014; Guerrero Ramos, 2013).

Los estudios de Fuentes et al. (2012) y Gerding et al. (2012, 2014) dan cuenta del rol fundamental que cumple la prensa en el español de Chile como vehículo de transvase de unidades léxicas anglicadas al español por la influencia de la cultura estadounidense. De hecho, estos estudios han determinado que la frecuencia de elementos anglicados en la prensa en español es alta y su recurrencia, creciente, y estos fenómenos redundarían en un proceso gradual de aceptación y adopción de estas voces por parte de los hablantes.

Normalmente, los anglicismos se introducen en una lengua en forma de préstamos y calcos. Siguiendo las proposiciones del OBNEO (2004), en el presente trabajo se define 'calco' como la unidad que corresponde a la traducción literal de una palabra o sintagma; 'préstamo', como la palabra de origen extranjero incorporada al español, con o sin adaptación ortográfica y, específicamente, 'préstamo del inglés', como la unidad 
de ese origen introducida al español. Teniendo estos conceptos en consideración, se analizan aquí específicamente dos subtipos de neologismos correspondientes a la formación por sintagmación: los sintagmas formados por calco anglicado y los sintagmas híbridos formados por préstamos del inglés y voces patrimoniales. Estas cadenas sintagmáticas corresponden a lo que el OBNEO (2004: 16) define como "unidades formadas por una estructura sintáctica lexicalizada".

Con respecto a los neologismos por formación híbrida, Estornell Pons (2012: 100) los define como piezas léxicas generalmente provenientes de una lengua de prestigio con influencia en determinados ámbitos y constituidas "por la combinación de un elemento patrimonial con otro de carácter foráneo". Entre los distintos mecanismos combinatorios, la autora destaca que el más frecuente es aquel formado por una voz patrimonial seguida un préstamo (por ejemplo, cine premium, contratar online, triple pack). Para este estudio el 'sintagma híbrido' es el que contiene al menos un elemento patrimonial (en español) en combinación con al menos un elemento anglicado en la cadena sintagmática. Ejemplos: 'música country', 'pie de manzana', 'crossfit al aire libre'. Los préstamos que forman parte de estas estructuras sintagmáticas pueden dividirse en léxicos y semánticos (Fischer, 2008). En el primer caso, se introduce de una lengua a otra una palabra junto con su significado (o parte de él), mientras que en el segundo, se introduce solo el significado de la palabra extranjera.

La noción de 'calco', según Gómez Capuz (2009), surge por oposición al préstamo. Este último corresponde a la transferencia integral de un elemento léxico extranjero, mientras que el calco siempre implica la idea de traducción y sustitución de morfemas. El calco es, entonces, la reproducción en la lengua receptora de un elemento extranjero cuya estructura o motivación semántica es similar a las del modelo foráneo. La imitación se realiza mediante un proceso sintético y con recursos de formación propios de la lengua que introduce el calco (Cabré, 2006; Gómez Capuz, 2009); por esta razón, a menudo pasa inadvertido para los hablantes. Ejemplos: 'estación de servicio' por service station, 'efecto invernadero' por greenhouse effect o 'economía social de mercado’ por social market economy.

Tal como se muestra en la Figura 1, existen varios tipos de calcos². Gómez Capuz (2009) plantea que el ‘calco léxico’ es el procedimiento de creación de unidades léxicas mediante la imitación de la estructura morfológica polimorfemática del modelo extranjero. Ejemplo: 'patio de comida(s)', 'patio gastronómico' o 'plaza de comida' por food court para referirse a la zona de restaurantes en un centro comercial. El autor propone varias subcategorías de calco léxico, de las cuales se recogen para este estudio el calco léxico literal (por ejemplo, 'enfermedad de las vacas locas', proveniente de mad cow disease) y el calco léxico aproximado (por ejemplo, 'cazatalentos', que se origina en beadhunter). 


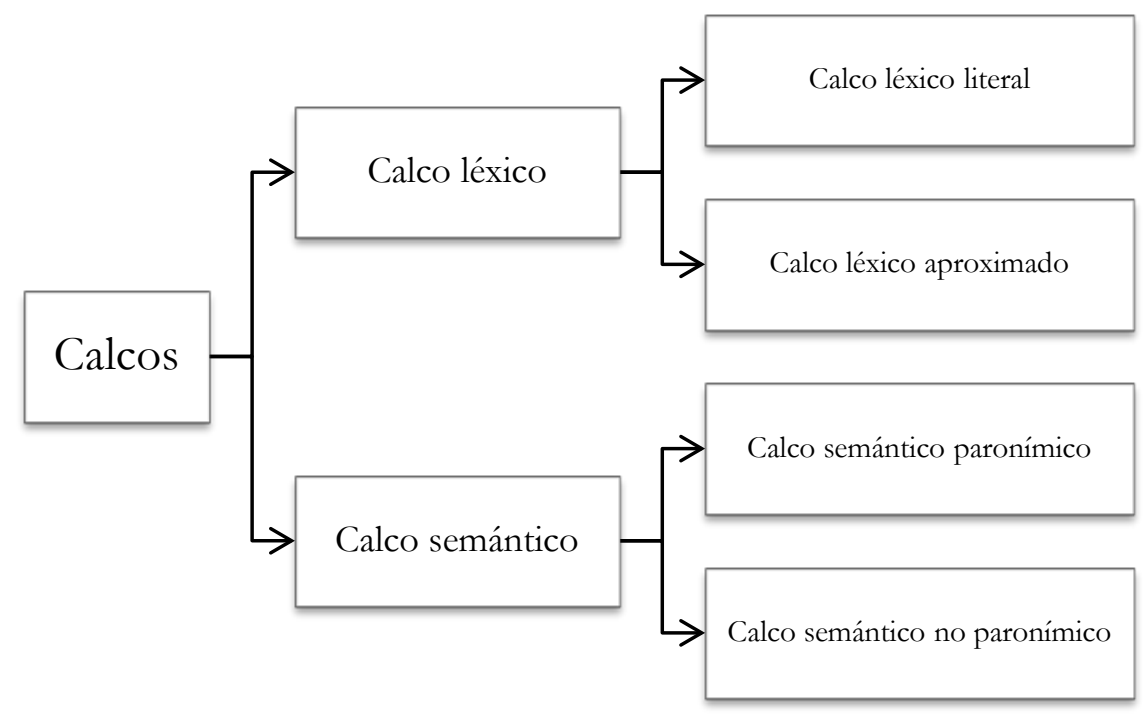

Figura 1. Esquema de los tipos de calcos considerados para este estudio.

A diferencia del calco léxico, el 'calco semántico' modifica el significado de una unidad ya existente en la lengua receptora. Por lo tanto, ocurre cuando el significado de una unidad léxica de una lengua determinada se agrega al significado de otra en la lengua receptora, por lo general a través de una extensión semántica (Gómez Capuz, 2009), que puede ser por analogía, ampliación o reducción del sentido original, aunque, según Cabré, Bayà, Bernal, Freixa, Solé y Vallès (2002) también se produce por cambio de significado. Ejemplo: 'plano, -na', proveniente de flat, con un nuevo sentido de 'fijo, -ja', como en el siguiente contexto: "Este viernes 8 de marzo comienza a regir la tarifa 'plana' entre diferentes operadores de telefonía móvil" (24horas.cl, 8 de marzo de 2013). Así, el calco semántico puede aportar una acepción total o parcialmente nueva a la lengua receptora. En cuanto a su identificación, por lo general, ante los calcos semánticos se presenta la incerteza de si la nueva acepción procede de un modelo extranjero o sencillamente del desarrollo semántico propio de una lengua, lo cual complejiza su detección y clasificación.

Los calcos semánticos, a su vez, pueden ser 'paronímicos' o 'no paronímicos'. Las unidades del primer tipo, llamadas también 'falsos amigos', presentan una similitud formal con palabras de otra lengua, parecido que facilita la transferencia semántica (Gómez Capuz, 2009). Ejemplo: se ha observado que 'dramático, -ca', cuyo significado en español es 'teatral, afectado o relativo al drama', se emplea de forma creciente con el sentido anglicado de 'drástico, -ca', como en el siguiente contexto: "Se aprobó un alza 'dramática' en el impuesto al barril de petróleo” (El Nuevo Día, 29 de abril de 2015). En las unidades del segundo tipo, en cambio, solamente existe una similitud conceptual entre palabras de dos lenguas, pero no formal ni etimológica. Por ejemplo: 'perro caliente' para hot dog. En pocas palabras, los calcos semánticos 
paronímicos son calcos de forma o imitaciones, mientras que los no paronímicos son calcos de significado o traducciones literales.

Tanto los calcos léxicos como los calcos semánticos suelen originarse y divulgarse por inercia o por una actitud negligente (Casas Tello, 1997; Gómez Capuz, 2009) y se observan con frecuencia en traducciones de textos técnicos, en seriales de televisión y en textos de agencias noticiosas. Dado el caudal de material que se traduce del inglés al español en estos y otros ámbitos, es frecuente encontrar calcos y préstamos anglicados en la prensa escrita en la actualidad.

Sobre la base de los planteamientos teóricos expuestos, este estudio tiene como objetivo general explorar la innovación léxica en unidades sintagmáticas con elementos anglicados detectadas en prensa escrita chilena. Específicamente, el trabajo pretende documentar las creaciones sintagmáticas anglicadas con el propósito de contribuir a la ampliación descriptiva de la lengua y aportar a la actualización lexicográfica.

\section{Metodología}

La recopilación de las unidades de estudio se realizó de forma manual y semiautomática, esta última mediante el Buscaneo del OBNEO. Esta herramienta extrae neologismos de prensa en línea de forma automática mediante el contraste de las unidades con el corpus lexicográfico de exclusión incorporado. Sin embargo, este mecanismo también recoge candidatos que no corresponden a unidades neológicas propiamente dichas, sino a otras formas, como algunos nombres propios, errores ortotipográficos, etc., los que el investigador debe eliminar.

Para su detección, se emplearon dos criterios de neologicidad, el psicolingüístico y el lexicográfico. El primero se refiere a lo que Sablayrolles (2003) denomina 'sentimiento de neologicidad', es decir, la sensación del hablante de estar ante una unidad nueva, y el segundo se refiere a la documentación de la unidad en diccionarios de lengua general, con lo que se descartan las unidades léxicas que aparecen como entrada o en los ejemplos de una entrada en alguno de los diccionarios establecidos como corpus lexicográfico de referencia.

En este estudio, el corpus de referencia estuvo constituido por las siguientes obras lexicográficas (ver Anexo 1):

- Diccionario de uso del español de América y España. Vox, Madrid, 2002.

- Diccionario de la lengua española. 23a edición, 2014, versión digital, Real Academia Española, Madrid, 2015. 
Las unidades sintagmáticas neológicas fueron relevadas de cuatro fuentes de vaciado, a saber: El Mercurio, El Sur, Las Últimas Noticias y The Clinic. Estos periódicos corresponden a publicaciones chilenas de circulación nacional y regional y a diversos niveles de registro lingüístico, lo que permite cautelar un espectro amplio de formas de expresión que se adoptan en función de las circunstancias. Anualmente se vacía un mínimo de 30 periódicos completos (excluyendo la publicidad), elegidos aleatoriamente.

El período de recopilación abarcó desde el 1 de enero de 2003 hasta el 31 de diciembre de 2014 y las unidades fueron extraídas de la base de datos de la Antena Chilena de Neología, Universidad de Concepción, que registra anualmente alrededor de 1.500 neologismos en fichas neológicas que contienen diversa información, por ejemplo: la fuente de relevo, la fecha de publicación, el autor de la ficha, la categoría gramatical del neologismo, el tipo de formación, el contexto, la sección y la página del periódico y un campo para notas especiales.

Los criterios utilizados para determinar el estatus de sintagma léxico híbrido o de sintagma por calco anglicado fueron la competencia lingüística de las investigadoras, es decir, su conocimiento de español e inglés, la intuición lingüística o impresión de estar ante una traducción literal o una anomalía, y la comprobación de la pertinencia del criterio intuitivo mediante documentación lexicográfica. Estos criterios coinciden con los que plantea Fischer (2008: 9), cuando se refiere a la dificultad que enfrenta el investigador al aplicar el criterio intuitivo y cómo puede resolverla:

“Another possibility is to base one's judgement on one's own instinctive feel for the native tongue, which, however, is subjective and therefore also a rather dubious method. The way out of this dilemma may be to check whether an anglicism is listed in a dictionary of foreign words of the language concerned, or whether it already occurs in a common monolingual desk dictionary."

Por lo demás, la intuición, cuya base es la experiencia, sirve para tener una aproximación inicial a un fenómeno: "También hay que entender la intuición no como un conocimiento autónomo, sino como un momento del proceso cognoscitivo" (Lara Castillo, 2004: 195) y es de carácter tentativo y provisional. De allí la necesidad de confirmar los hallazgos realizados intuitivamente con métodos objetivables, como ocurre en el proceso de identificación de neologismos.

El corpus de referencia en inglés en línea, que se utilizó determinar la existencia de las unidades sintagmáticas en inglés $\mathrm{y}$, con ello, establecer etimológicamente su procedencia o, en su defecto, inferir su origen, se empleó también para la comprobación de los significados de los sintagmas anglicados o de nuevas acepciones de alguno de sus elementos constituyentes. Este corpus referencial fue el siguiente (ver Anexo 1): 
- Merriam-Webster, como referente monolingüe de 'inglés estadounidense'

- Oxford Dictionaries, como referentes monolingües de 'inglés británico'

- WordReference como referente bilingüe español-inglés e inglés-español, actualizable y con foro de discusión

- Enciclopedias colaborativas y otra documentación en internet, en caso de léxico de muy reciente aparición o de unidades terminológicas.

De este modo, del total de 817 unidades sintagmáticas de diversos tipos registradas en la base de datos de la Antena Chilena de Neología durante el período de investigación, 190 unidades resultaron ser sintagmas anglicados, lo que representa un $23 \%$.

\section{Análisis y discusión de resultados}

Las 190 unidades que constituyeron la muestra correspondieron a 26 sintagmas híbridos con préstamo anglicado (14\%) y 164 sintagmas formados por calco anglicado (86\%). Una razón que podría explicar la baja cifra de sintagmas híbridos es que en muchos casos el elemento anglicado ya formaba parte del corpus lexicográfico de exclusión, razón por la que esta unidad pierde su condición de híbrido (por ejemplo: 'cultura pop'), a diferencia de sintagmas como bullying virtual, cuyo elemento anglicado no está registrado en el corpus de referencia.

Al contrastarse los sintagmas con el corpus de exclusión del español de Chile (Nuevo diccionario ejemplificado de chilenismos y de otros usos diferenciales del español de Chile, 2010), se determinó que 164 unidades (86\%) eran neológicas para esta variante. De las 26 unidades no neológicas, 25 estaban formadas por calco anglicado ('tarifa plana', 'lavado de dinero') y solo una por hibridación con préstamo del inglés ('a full'). Se constató mediante búsquedas en internet que algunos de los sintagmas híbridos tenían un equivalente en español, por lo general en la forma de calco; es probable que la preferencia por el uso anglicado en la fuente de vaciado obedeciera a un cierto grado de esnobismo o a una moda, como por ejemplo, 'teléfono smart' en lugar de 'teléfono inteligente'. Llamó la atención que en el español de Chile persistiera el uso de algunas unidades sintagmáticas formadas con un préstamo integral, aunque ya se hubiera acuñado un equivalente en español por la vía de la traducción literal; por ejemplo: 'televisor smart', en lugar de 'televisor inteligente', 'horario prime', en lugar de 'horario estelar' o 'red wifi', en lugar de 'red inalámbrica'. Este tipo de formación es poco recurrente, pero las unidades creadas de este modo se han mantenido en el tiempo, como lo corroboran las fechas de las fichas neológicas incluidas en el estudio. Es probable que las razones de los hablantes para usar estas unidades obedezcan a economía lingüística, a un sello de prestigio o moda y que algunas de ellas hayan sido introducidas a la lengua por los medios de comunicación o 
por la publicidad en el proceso de desterminologización de unidades pertenecientes a los lenguajes de especialidad, como ocurre en el ámbito de la tecnología, por ejemplo.

Por otra parte, de los sintagmas formados mediante el mecanismo de traducción literal, los calcos léxicos fueron muy superiores en número a los calcos semánticos (ver Tabla 1). Es probable que el mayor número de sintagmas formados por calco léxico se deba a que existe una mayor facilidad de detección para el neólogo, en comparación con los calcos semánticos, para cuya localización se requiere un análisis mucho más profundo por no hacerse evidentes a simple vista del recopilador. Entre los calcos léxicos predominaron los de tipo literal, como 'cientista político', proveniente de political scientist, lo que confirmaría que este tipo de unidades se suele formar mediante la traducción palabra por palabra. Por otra parte, no se observó una diferencia cuantitativamente importante entre los calcos semánticos paronímicos y los no paronímicos, los que en conjunto alcanzaron un porcentaje inferior al $20 \%$, como se observa en la Tabla 1.

Tabla 1. Tipos de calcos léxicos y recurrencia.

\begin{tabular}{|l|c|c|c|}
\hline \multicolumn{1}{|c|}{ Tipo de calco } & Recurrencia & Porcentaje & Porcentaje acumulado \\
\hline Calcos léxicos literales & 131 & 79,87 & 79,87 \\
\hline Calcos léxicos aproximados & 4 & 2,44 & 82,31 \\
\hline Calcos semánticos no paronímicos & 17 & 10,37 & 92,68 \\
\hline Calcos semánticos paronímicos & 12 & 7,32 & 100,00 \\
\hline Total & 164 & 100,00 & \\
\hline
\end{tabular}

A continuación se ilustran las distintas categorías de sintagmas formados por calco:

Calco léxico literal: 'dispositivo vestible', de wearable device, y 'profecía autocumplida', de self-fulfilling prophecy. En el primer sintagma, el elemento 'vestible' también es neológico y en el segundo se observa una influencia terminológica desde la sociología en el uso actual de la unidad. Estos ejemplos confirmarían el papel que cumplen los medios de comunicación en la creación neológica, ya sea debido a la traducción literal a la que obligaría la premura por publicar o a la desterminologización de unidades especializadas.

Calco léxico aproximado: 'píldora del día después', de morning after pill (que presenta una adaptación del elemento morning por 'día') y 'anticonceptivo de emergencia', de emergency contraceptive pill (que presenta la elisión del elemento 'píldora' al usarse 'anticonceptivo' en forma nominal). Una posible razón de estas adaptaciones obedecería a diferencias culturales asociadas a cada una de las lenguas, lo que explicaría la elección de 'día' por morning, y en el caso de 'anticonceptivo' por contraceptive pill, se evidencia un acortamiento por economía lingüística.

Calco semántico paronímico: fueron recurrentes los sintagmas formados con el elemento 'orgánico, ca', en el sentido del inglés not using artificial chemicals (MerriamWebster (2016) en Anexo 1) como 'vino orgánico', 'viña orgánica', 'huerto orgánico’, 
'café orgánico. En estos casos, el parecido de la voz en ambas lenguas facilita la transferencia del significante y la adquisición de un nuevo significado. El adyacente 'orgánico', que es neológico en español con la acepción del inglés ('sin sustancias químicas artificiales'), resultó ser uno de los elementos más productivos en la formación de sintagmas de este estudio.

Calco semántico no paronímico: en los ejemplos 'dinero blando', 'habilidades blandas' y 'competencias blandas', provenientes de soft money, soft skills y soft competences, respectivamente, se observa también el traslado del significante y la adquisición de un nuevo significado, donde el adyacente 'blando' adopta en español nuevas acepciones que porta el elemento soft en estas combinaciones sintagmáticas en inglés. En el caso de 'dinero blando' el adyacente se refiere a 'fondos deducibles de impuestos, donados a partidos políticos' y en los otros dos ejemplos, a 'rasgos de personalidad o de conducta'. Probablemente lo breve del adyacente soft invita a su adopción con el sentido del inglés de 'aquello fundado en los valores y las emociones'.

En cuanto a las estructuras que forman los sintagmas, a continuación se presenta, en primer lugar, el análisis de los sintagmas neológicos creados por formación híbrida $y$, en segundo, el de los calcos. Del total de neologismos híbridos, el 80\% correspondió a sintagmas nominales, lo que coincide con otros estudios sobre préstamos del inglés con respecto a la recurrencia de nombres (Gerding et al., 2012; Gerding et al., 2014); el 20\% restante estuvo constituido por sintagmas verbales y preposicionales. Seguidamente se presentan las cadenas sintagmáticas de mayor frecuencia, con ejemplos. La explicitación de las abreviaturas de cada estructura se encuentra en el Anexo 2 al final de este artículo:

Tipo de sintagma y estructura

$\mathrm{SN}[\mathrm{N}+$ AdjAngl]

$\mathrm{SN}$ [NAngl+Adi]

$\mathrm{SN}$ [N+NAngl fn Adj]

$\mathrm{SV}[\mathrm{V}+\mathrm{Nangl}]$

SP [Prep+NAngl]

\section{Ejemplos}

'secuestro express', 'horario peak'

'bullying virtual', 'team nuclear'

'chico reality', 'teléfono touch'

'quedar plop' (quedar atónito)

'a finish'

La estructura sintagmática con mayor presencia en la muestra fue $\mathrm{SN}$ [N+NAng] fn Adj] con un $40 \%$.

En relación con los calcos neológicos, la gran mayoría estuvo constituida por sintagmas nominales de dos tipos: 
Tipo de sintagma y estructura

$$
\mathrm{SN}[\mathrm{N}+\operatorname{Adj} 1+(\operatorname{Adj} 2)]
$$

$\mathrm{SN}[\mathrm{N}+\operatorname{Prep}+($ Art $)+\mathrm{N}]$

\section{Ejemplos}

'contaminación cruzada', de crosscontamination

'responsabilidad social empresarial', de corporate social responsibility

'tienda de conveniencia', de convenience store ‘cese al fuego' (sus variantes 'alto al fuego' y 'cese del fuego' no son neológicas), de ceasefire o cease-fire

Prácticamente $53 \%$ de los sintagmas por calco correspondieron a la primera estructura, mientras que 31\% correspondió a la segunda. La fuerte presencia de estas dos estructuras sintagmáticas anglicadas deja de manifiesto la traducción literal, en la que se suele respetar el genio de la lengua meta en el traslado.

Entre las estructuras sintagmáticas de menor representación (con menos de $2 \%$ cada una) se encontraron sintagmas verbales, adverbiales y preposicionales, de los cuales se presentan ejemplos a continuación:

Tipo de sintagma y estructura

$\mathrm{SV}[\mathrm{V}+($ Art $)+\mathrm{N}]$

SV $[\mathrm{V}+\operatorname{Pron}+\mathrm{Adj}]$

$\mathrm{SAdv}[\mathrm{Adv}+\mathrm{N}]$

SP [Prep+N+Adj]

\section{Ejemplos}

'hacer la diferencia' por 'marcar la diferencia' 'hágalo usted mismo' por 'ármelo usted' 'como seda' por 'suave, dócil' 'de clase mundial' por 'de primera categoría'

Algunos sintagmas presentaron variantes denominativas, como 'a tajo abierto' y 'de tajo abierto', provenientes de open cut, y 'patio de comidas', 'plaza de comida' y 'patio gastronómico', de food court. En el primer caso, posiblemente se trate de la preferencia del hablante frente a la unidad lexicográficamente documentada 'de rajo abierto' y, en el segundo, la elección de una u otra variante estaría determinada por el registro lingüístico asociado a determinado estatus social, pero no a una fuente de relevo específica, aunque en todos los casos se trata evidentemente de traducciones más o menos literales.

Por otra parte, se detectaron adyacentes con una frecuencia bastante alta, entre los que sobresalen dos: 'inteligente' con 8 ocurrencias y 'digital' con 9 ocurrencias.

Por ejemplo:

- 'alfabetización digital', 'brecha digital', 'firma digital', 'nativo digital'

- 'casa inteligente', 'vestuario inteligente', 'calcetines inteligentes' 
Es importante señalar que todos estos sintagmas son neológicos; sin embargo, ambos adyacentes ya se encuentran documentados lexicográficamente en el corpus de exclusión con las acepciones portadas desde el inglés.

Con respecto a las áreas temáticas en las que se encontraron sintagmas anglicados, se observó que algunas de éstas correspondían a secciones específicas del periódico, mientras que otras son transversales a varias secciones. A continuación se presentan ejemplos de sintagmas neológicos anglicados recogidos de las áreas más productivas, de las cuales las tres primeras representan prácticamente el 50\% (18,3\%, 8,5\%, 17,3\%, respectivamente):

- Tecnología e informática: ‘código abierto', de open code, ‘pantalla touch', de touch screen

- Medicina y salud: 'helicóptero medicalizado', de medicalized helicopter, 'período (de) ventana' (período entre la infección y la detección), de window period

- Economía y comercio: 'costo hundido' (costo no recuperable), de sunk cost, 'fondo buitre' (fondo de capital de riesgo), de vulture fund

- Medioambiente: 'producción limpia', de clean production, 'gas de invernadero', de greenhouse gas

- Derecho: 'violencia de género', de gender violence, 'adopción plena', de full adoption

La alta frecuencia de sintagmas neológicos por calco del inglés en estos ámbitos se debería, en gran parte, a los avances tecnológicos introducidos al país, a la influencia sociocultural de Estados Unidos y a la adopción de paradigmas económicos, todos ellos divulgados a través de la prensa. De la observación espontánea habitual se puede colegir, además, que este tipo de neologismos es muy frecuente en la publicidad, como también lo avalan algunos estudios (Hooft, 2016; Valozic, 2015).

Por último, se observó un escaso uso de marcas tipográficas para indicar la neologicidad de los sintagmas. Dado que los manuales de estilo por lo general recomiendan el uso de letra cursiva o de comillas para marcar unidades provenientes de otras lenguas o para unidades neológicas, se esperaba encontrar un mayor número de sintagmas destacados de esta forma. La presencia de estas marcas podría eventualmente alertar al lector con respecto a la presencia de estas unidades (Freixa, 2010), quedando la intelección supeditada a su propio conocimiento.

\section{CONCLUSIONES}

En relación con la motivación de los hablantes para usar sintagmas neológicos por calco o préstamo anglicados, este estudio permite suponer que este tipo de innovación no sólo obedece a la necesidad de cubrir vacíos denominativos, sino también a cuestiones de índole estilística. Si bien el léxico neológico anglicado es recurrente en la 
denominación de nuevos referentes en el español de hoy, también puede desempeñar otras funciones en la prensa: aportar espontaneidad al texto, enfatizar algunos significados específicos o, eventualmente, comunicar con eficacia.

En cuanto a los mecanismos mediante los cuales se acuñan estas piezas léxicas, la mayoría de los sintagmas detectados evidenció el traspaso literal del inglés al español, cuestión que probablemente se origina en lo diligente y oportuna que se espera sea la comunicación, aunque también se podría atribuir a una cierta esterilidad creativa o simple desconocimiento lingüístico por parte de quienes introducen las unidades léxicas extranjeras a la lengua patrimonial. Es el caso recurrente de 'cientista político' en la prensa, cuyo uso desatiende la existencia de la voz 'politólogo' registrada en los diccionarios. Las combinaciones sintagmáticas híbridas, por otra parte, se emplearían en gran medida por razones de prestigio o esnobismo, especialmente cuando persiste el uso de la unidad híbrida con un elemento anglicado y aun cuando su equivalente se haya documentado lexicográficamente. En efecto, la alta frecuencia de calcos léxicos literales estaría relacionada con traducciones apresuradas y poco reflexivas que no pocas veces conducen a usos difíciles de erradicar de voces que terminan imponiéndose.

La presencia de la lengua y la cultura anglosajonas en la variedad chilena del español es evidente, como lo demuestra la formación de unidades sintagmáticas con elementos anglicados por la vía del préstamo o la traducción literal desde el inglés. La proporción de sintagmas anglicados respecto de toda la formación sintagmática neológica de la base de datos de la Antena Chilena de Neología, Universidad de Concepción, confirma resultados de estudios anteriores que dan cuenta de la gravitación de los préstamos entre los mecanismos de mayor peso en la innovación léxica del español actual.

Por otra parte, a partir de los hallazgos de este estudio se validaría la necesidad de una mayor inclusión de sintagmas lexicalizados en la actualización lexicográfica. Para ello sería esencial establecer criterios cada vez mejor definidos para determinar los tipos de sintagmas que deberían figurar en los diccionarios. En ese sentido, este trabajo podría ser una contribución a la discusión neológica. Finalmente, indagar acerca de las motivaciones que impelen a los periodistas a usar voces anglicadas y calcos sería un ejercicio revelador que valdría la pena realizar.

\section{REFERENCIAS BIBLIOGRÁFICAS}

Alvar Ezquerra, M. (1998). Palabras nuevas en los periódicos de hoy. En A. Álvarez Tejedor (Ed.), La lengua española a finales del milenio (pp. 13-44). Burgos: Caja de Burgos. 
Alvar Ezquerra, M. (1999). El neologismo: Caracterización, formación y aceptabilidad. En J. M. González Calvo, Mª L. Montero Curiel \& J. Terrón González (Eds.), Actas V Jornadas de Metodología y Didáctica de la Lengua Española: El neologismo (pp. 39-66). Cáceres: Universidad de Extremadura.

Alvar Ezquerra, M. (2007). El neologismo español actual. En L. Luque Toro (Ed.), Léxico Español Actual, Actas del I Congreso Internacional de Léxico Español Actual (pp. 11-35). Venecia: Librería Editrice Cafoscarina.

Arroyo, A. \& Sevilla, J. (1993). La noción de expresión idiomática en francés y en español. Revista de Filología Francesa, 4, 247-261.

Bernal-Meza, R. (2008). Argentina y Brasil en la política internacional: Regionalismo y Mercosur (estrategias, cooperación y factores de tensión). Revista Brasileira de Política Internacional, 52(2), 154-178.

Briceño Ruiz, J. (2006). Regionalismo estratégico en las relaciones de la Unión Europea con América del Sur. Comercio Exterior, 56(5), 428-434.

Cabré, M. T. (1993). La Terminología. Teoría, metodología y aplicaciones. Barcelona: Editorial Antártida/Empúries.

Cabré, M. T. (2006). La clasificación de neologismos: Una tarea compleja. Alfa, 50(2), $229-250$.

Cabré, M. T., Bayà, M. R., Bernal E., Freixa, J., Solé, E. \& Vallès, T. (2002). Evaluación de la vitalidad de una lengua a través de la neología: A propósito de la neología espontánea y de la neología planificada. En M. T. Cabré, J. Freixa \& E. Solé (Eds.), Lexic i Neologia (pp. 159-201). Barcelona: Observatori de Neologia, Institut Universitari de Lingüística Aplicada, Universitat Pompeu Fabra.

Casas Tello, S. (1997). Calcos lingüisticos y fraseológicos en el lenguaje audiovisual: El caso de Pulp Fiction [en línea]. Disponible en: http://www.uji.es/bin/publ/edicions/jfi3/calcos.pdf

Delgado Álvarez, A. (2005). Los anglicismos en la prensa escrita costarricense. Káñina, 29 (Número especial), 89-99.

Deroy, L. (1971). Néologie et néologismes: Essai de typologie générale. La Banque des Mots, 1, 5-12.

Diéguez, M. I. (2004). El anglicismo léxico en el discurso económico de divulgación científica del español de Chile. Onomázęin, 2(10), 117-141. 
Domínguez Mejías, E. (2002). Los anglicismos en el diccionario de la RAE (2001). Panace@,3(8),28-33.

Esteban Asencio, L. (2008). Uso, origen y procesos de creación de neologismos en prensa española. Círculo de lingüistica aplicada a la comunicación, 33, 3-27.

Estornell Pons, M. (2012). Novedades léxicas en revistas femeninas: Procedimientos de formación y valor semántico-pragmático de las unidades. Normas. Revista de Estudios Lingüisticos Hispánicos, 2, 77-108.

Fischer, R. (2008). Introduction. Studying anglicisms. En R. Fischer \& H. Pulaczewska (Eds.), Anglicisms in Europe: Linguistic Diversity in a Global Context (pp. 1-15). Newcastle upon Tyne: Cambridge Scholars Publishing.

Freixa, J. (2010). La neologicidad en las unidades formadas por prefijación. Puente, 9, $11-32$.

Fuentes, M., Gerding, C., Gómez, L. \& Kotz, G. (2012). El préstamo en seis variedades geolectales del español: Un estudio en prensa escrita. Revista Signos. Estudios de Lingüistica, 40(80), 281-299.

Gerding, C., Fuentes, M. \& Kotz, G. (2012). Anglicismos y aculturación en la sociedad chilena. Onomázein, 25(1), 139-162.

Gerding, C., Fuentes, M., Gómez, L. \& Kotz, G. (2014). Anglicisms: An active wordformation mechanism in Spanish. Colombian Applied Linguistics Journal, 16(1), 40-54.

Gimeno, F. \& Gimeno, M. V. (2003). El desplazamiento lingüistico del español por el inglés. Madrid, Cátedra.

Goddard, K. A. (1980). Loan-words in Spanish. A reappraisal. Bulletin of Hispanic Studies, LVII, 1-16.

Gómez Capuz, J. (2009). El tratamiento del préstamo lingüístico y el calco en los libros de texto de bachillerato y en las obras divulgativas. Tonos. Revista electrónica de estudios filológicos, 17.

Guerrero Ramos, G. (2013). El préstamo lingüístico, uno de los principales procedimientos de creación neológica. Quaderns de Filologia. Estudis lingüistics, 18, 115-130.

Hooft, A. van (2016). El valor añadido de los anglicismos en la publicidad española. El impacto y la valoración social de las voces inglesas en los anuncios de la revista Elle. En M. V. Calvi \& L. Chierichetti (Eds.), Nuevas tendencias en el discurso de especialidad (pp. 219-243). Frankfurt am Main: Peter Lang. 
Lara Castillo, R. (2004). Intuición y conocimiento. Umbral. Revista de Educación, Cultura y Sociedad, IV (7), 194-195.

Lorenzo, E. (1996). Anglicismos hispánicos. Madrid, Gredos.

Miletich, M. (2006). Pedagogía del error: Aspectos didácticos de la traducción. En Centro Virtual Cervantes (pp. 453-465) [en línea]. Disponible en: http://cvc.cervantes.es/lengua/esletra/pdf/03/041_miletich.pdf.

Morin, R. (2006). Evidence in the Spanish language press of linguistic borrowings of computer and Internet-related terms. Spanish in Context, 3(2), 161-179.

Mounin, G. (1990). Quelques observations sur le lexique français d'aujourd'hui. Europe, 738, 10-18.

Novotná, M. (2007). El anglicismo en la lengua española. Tesis de Licenciatura, Universidad de Masaryk, Brno, República Checa.

Observatori de Neologia (2004). Papers de l'IULA, Sèrie Monografies, 9. Metodología del trabajo en neología: Criterios, materiales y procesos. Universitat Pompeu Fabra, Institut Universitari de Lingüistica Aplicada, Barcelona.

Oncins-Martínez, J. L. (2009). Towards a corpus-based analysis of anglicisms in Spanish: A case study. International Journal of English Studies, número especial, 116-132.

Ortega Martín, M.P. (2001). Neología y prensa: Un binomio eficaz. Espéculo. Revista de estudios literarios, $18, \mathrm{~s} / \mathrm{p}$.

Perdiguero, H. (2003). Innovación léxica en la prensa. En H. Perdiguero \& A. Álvarez (Eds.), Actas del XIV Congreso Internacional de ASELE (pp. 88-95). Burgos: Servicio de Publicaciones Universidad de Burgos.

Romero Gualda, M. V. (1999). Neologismo y medios de comunicación. En J. M. González Calvo, Ma L. Montero Curiel \& J. Terrón González (Eds.), Actas V Jornadas de Metodología y Didáctica de la Lengua Española: El neologismo (pp. 67-96). Cáceres: Universidad de Extremadura.

Sáez Godoy, L. (2005). Anglicismos en el español de Chile. Atenea, 492, 171-177.

Sablayrolles, J. F. (2003). L’innovation lexicale. París: Honoré Champion.

Valozic, L. (2015). Anglicismo léxico en la publicidad. Alicante: Publicacions de la Universitat d'Alacant. 


\section{NOTAS}

${ }^{1}$ Proyecto de la Dirección de Investigación de la Universidad de Concepción, Chile, código 03.F5.01.

2 Arroyo y Sevilla (1993) y Miletich (2006) identifican varias clases, entre los que se incluyen los sintácticos, fraseológicos e idiomáticos, pero que no se han considerado en este estudio por no ser atingentes a él.

\section{ANEXO 1}

\section{Diccionarios de español}

- Diccionario de uso del español de América y España. Vox, Madrid, 2002.

- Diccionario de la lengua española. 23a edición, 2014, versión digital, Real Academia Española, Madrid, 2015.

- Nuevo diccionario ejemplificado de chilenismos y de otros usos diferenciales del español de Chile. Félix Morales Pettorino. Editorial Puntángeles, Valparaíso, 2010.

\section{Diccionarios de inglés}

- Merriam-Webster Dictionary and Thesaurus (http://www.merriam-webster.com/)

- Oxford Dictionaries, Thesaurus \& Grammar (http://www.oxforddictionaries.com/)

- WordReference (http://www.wordreference.com/)

\section{ANEXO 2}

En el análisis de resultados se emplean las siguientes abreviaturas para representar las estructuras gramaticales que forman las distintas combinaciones sintagmáticas:

- $\mathrm{SAdv}=$ sintagma adverbial

- $\mathrm{SN}=$ sintagma nominal

- $\mathrm{SP}=$ sintagma preposicional

- $\mathrm{SV}=$ sintagma verbal

- $\quad$ NAngl+Adj]: = nombre anglicado + adjetivo

- $\quad[\mathrm{N}+\mathrm{NAngl} \mathrm{fn}$ Adj $]=$ nombre anglicado con función de adjetivo

- $[\mathrm{V}+\mathrm{Nangl}]=$ verbo + nombre anglicado

- $\quad[$ Prep+NAngl $]=$ preposición + nombre anglicado

- $[\mathrm{N}+$ Adj1 $+($ Adj2 $)]=$ nombre + adjetivo $1+$ adjetivo 2

- $[\mathrm{N}+\operatorname{Prep}+($ Art $)+\mathrm{N}]=$ nombre + preposición $+($ artículo $)+$ nombre

- $\quad[\mathrm{N}+$ AdjAngl $]=$ nombre + adjetivo anglicado

- $[\mathrm{V}+($ Art $)+\mathrm{N}]=$ verbo $+($ artículo $)+$ nombre

- $[\mathrm{V}+$ Pron $+\mathrm{Adj}]=$ verbo + pronombre + adjetivo

- $[\operatorname{Adv}+\mathrm{N}]=$ adverbio + nombre

- $\quad[$ Prep $+\mathrm{N}+$ Adj $]=$ preposición + nombre + adjetivo 